\title{
Long non-coding RNA XIST accelerates hepatic carcinoma progression by targeting the microRNA-320a/PIK3CA axis
}

\author{
LINA YANG, FANGLIANG XIE, WEIDONG XU, TONGLEI XU, YUAN NI, XIAO TAO, YU ZANG and JUAN JIN \\ Department of Hepatobiliary Surgery, Lianyungang No. 1 People's Hospital, Lianyungang, Jiangsu 222002, P.R. China
}

Received September 15, 2020; Accepted July 27, 2021

DOI: 10.3892/ol.2021.13062

\begin{abstract}
The aim of the present study was to reveal the new molecular mechanism of long non-coding (lnc)RNA XIST in the development of hepatic carcinoma. A total of 69 patients with hepatic carcinoma were included. Hepatoma cell lines (SUN449), hepatoblastoma cell line (HepG2, Huh-6), liver cancer cell line (HepG2) and transformed human liver epithelial-2 cells (THLE-2) were used in the present study. A total 3 short hairpin RNA (sh)-lncRNA XIST sequences, overexpression vector (oe)-lncRNA XIST, microRNA (miR)-320a mimic, miR-320a inhibitor, PIK3CA inhibitor, and their corresponding controls were transfected in hepatic carcinoma cells. Reverse transcription-quantitative polymerase chain reaction was conducted to detect IncRNA-XIST, miR-320a and PIK3CA expression. Cell Counting Kit- 8 assay and flow cytometry were undertaken to measure proliferation and apoptosis. Cell invasion and migration were detected by Transwell assays. Moreover, the binding of lncRNA XIST, PIK3CA and miR-320a were verified by luciferase reporter experiment and pull-down assay. Finally, a rescue assay was processed to confirm the effect of IncRNA-XIST, miR-320a and PIK3CA in the aforementioned processes. IncRNA XIST was highly expressed in hepatic carcinoma tissues and cells. The survival rate was significantly lower in the highly expressed lncRNA XIST group. shlncRNA XIST attenuated cell proliferation, invasion and migration, while increasing the apoptosis of hepatic carcinoma cells. The lncRNA XIST negatively targeted miR-320a, and miR-320a negatively regulated the expression of PIK3CA. The miR-320a mimic and PIK3CA inhibitor could recover the effect of oe-lncRNA in terms of the proliferation, invasion, migration and apoptosis of hepatic carcinoma cells. IncRNA XIST accelerates hepatic carcinoma progression by targeting the miR-320a/PIK3CA axis, which
\end{abstract}

Correspondence to: Dr Juan Jin, Department of Hepatobiliary Surgery, Lianyungang No. 1 People's Hospital, 6 Zhenhua East Road, Haizhou, Lianyungang, Jiangsu 222002, P.R. China E-mail: jinjuan1975@163.com

Key words: long non-coding RNA XIST, microRNA-320a, PIK3CA, hepatic carcinoma, prognosis might provide the theoretical basis for the potential targeted therapy of hepatic carcinomas.

\section{Introduction}

Hepatic carcinomas are among the most common malignant tumors in China, and cause serious threat to individuals' health (1). The fatality rate of Hepatic carcinoma ranks second among malignant tumors. At present, surgical resection and liver transplantation are effective treatments for early hepatic carcinoma (2). With advancements in research on various surgical and drug treatments, the 5-year survival rate of hepatic carcinoma patients has increased (3). However, advanced stage hepatic carcinoma lacks effective treatment and intervention, and it is prone to metastasis at the early stages (4). During the first clinical visit, most patients with hepatic carcinoma already have micrometastases; the various complex molecular mechanisms in the occurrence and development of liver cancer are still unclear (5). Clinically, there is an urgent need to identify the effective molecular biological markers for the treatment and prognosis of hepatic carcinoma.

Long non-coding (lnc)RNAs play important roles in the development, differentiation, proliferation and apoptosis of cells $(6,7)$. For instance, Deng et al (8) compared 66 pairs of hepatic carcinoma tissues and normal tissues and found that CCAT1 expression was increased in liver cancer tissues, and that the degree of upregulation was associated with tumor size, vascular invasion and serum alpha-fetoprotein level. Further, Zamani et al (9) investigated the epigenetic effects of solute carrier family 25 member 19 in hepatic carcinoma and confirmed that DNA methylation in the functional region of maternally expressed 3 (MEG3) was associated with its loss of expression in hepatic carcinoma. Interestingly, ZNFX1 antisense RNA 1 (ZFAS1) could abolish its tumor suppressor effect by binding microRNA (miR)-150, while miR-150 could inhibit the invasion of hepatic carcinoma cells by inhibiting zinc finger E-box binding homeobox 1 (ZEB1) and matrix metalloproteinases (MMP)14 and MMP16 (10). H19 was also found to induce p-glycoprotein expression and MDR1-associated drug resistance in hepatic carcinoma cells by regulating the demethylation of the MDR1 promoter (11). In this way, the discovery of the tumor-associated protein lncRNA and elucidation of its associated mechanisms will provide new insights in terms of the prognosis and treatment of hepatic carcinoma. 
As reported in previous studies, IncRNA XIST plays the role of an oncogene in most tumors, the level of which was associated with short survival and poor prognosis $(12,13)$. In cases of breast cancer, patients with the low expression of lncRNA XIST were more sensitive to chemotherapy upon receiving high doses of alkylating agents, suggesting that this protein can be used as a biomolecular marker for breast cancer (14). Furthermore, the highly expressed lncRNA XIST promoted the cell proliferation and invasion of pancreatic cancer cells by negatively regulating miR-34a-5p expression. In addition, it was also found that the regulatory mechanism of XIST in lung cancer was associated with the activation of the enhancer Of zeste 2 polycomb repressive complex 2 subunit, leading to the silencing of Kruppel-like factor 2 (KLF2) (15). Various studies have focused on the role of lncRNA XIST in the development of hepatic carcinoma $(16,17)$, but the associated molecular mechanism was complicated. In the present study, the molecular mechanism of lncRNA XIST in the development of hepatic carcinoma was explored; moreover, the regulated association of lncRNA XIST/miR-320a/PIK3CA was also shown. This might provide a new experimental basis for further revealing the association between lncRNA XIST and the associated biomarkers of hepatic carcinoma.

\section{Materials and methods}

Patients and tissues. The samples were obtained from the surgical tissues of 69 patients with hepatic carcinoma at the Lianyungang First People's Hospital. Samples $>3 \mathrm{~cm}$ from the edge of the cancerous tissue were used as the adjacent-cancerous tissues. All patients signed an informed consent form approved by the Lianyungang No. 1 People's Hospital ethics committee (permit no. LW-20140322001), which complied with medical ethics regulations. Of the enrolled patients, 39 were male and 30 were female. The patients' age ranged from 20-71 years, with a median age of 52 years. None of the patients received adjuvant treatments such as radiotherapy, chemotherapy or radiofrequency ablation, and the postoperative specimens were confirmed to be hepatocellular carcinoma by pathological examination. The specimens of the control group were adjacent tissues located at least $3 \mathrm{~cm}$ from the surgical margin, and no cancer cells were found on postoperative pathological examination. All specimens were quickly frozen in liquid nitrogen after being obtained and stored in a refrigerator at $-80^{\circ} \mathrm{C}$.

Cell culture and transfection. Hepatoma cell line (SUN449), hepatoblastoma cell line (Huh-6), liver cancer cell line (HepG2) and transformed human liver epithelial-2 cells (normal hepatocytes; THLE-2) were purchased from the American Type Culture Collection. The short tandem repeat method was used for authentication. The cells were cultured in Dulbecco's Modified Eagle's Medium (DMEM) containing 10\% fetal calf serum (both Invitrogen; Thermo Fisher Scientific, Inc.) and placed in an incubator at $37^{\circ} \mathrm{C}$ containing $5 \% \mathrm{CO}_{2}$. After $0.25 \%$ trypsin digestion, the cells were passaged, and the cells in the logarithmic growth phase were used for the experiments.

A total of 3 short hairpin RNA (sh)-lncRNA XIST sequences, overexpression vector (oe)-lncRNA XIST, miR-320a mimic, miR-320a inhibitor, PIK3CA inhibitor, and their corresponding controls were designed and synthesized by IBSBIO Co. Ltd. Cells were co-transfected with shlncRNA XIST ( $2 \mu \mathrm{g}$; Ibsbio Co., Ltd.) and the viral packaging plasmid $(2 \mu \mathrm{g})$ (Nanjing Cobioer Gene Technology Co., Ltd.) The expression was detected after incubation at $37^{\circ} \mathrm{C}$ for 72-96 h. After 72 h, G418 was added for screening. After 48 h of transfection, G418 was added to each group to achieve a final concentration of $0,200,400,600,800$ and $1,000 \mathrm{mg} \cdot \mathrm{l}^{-1}$, respectively. After continuous observation for 4-7 days, the G418 concentration, which can lead to complete cell death in the untransfected group, was selected as the optimal working concentration for the subsequent screening of transfected cells. According to the selected optimal G418 concentration, the transfected cells were maintained in the culture until a stable viable cell line was obtained. Lipofectamine ${ }^{\circledR} 2000$ reagent (Thermo Fisher Scientific, Inc.) was also used to transfect oe-lncRNAXIST. The corresponding empty vector was used as a control. Similarly, the miR-320a mimic, miR-320a inhibitor, PIK3CA inhibitor, and their corresponding controls were also transfected by Lipofectamine 2000 at $37^{\circ} \mathrm{C}$, as aforementioned. Non-targeting sequence was used as shNC, inhibitor NC, siNC and NC for negative control experiments. A non-targeting sequence was the negative control for transfections of miR-320a mimic. After 24 or $48 \mathrm{~h}$, the following experiments were conducted.

Reverse-transcription-quantitative polymerase chain reaction $(R T-q P C R)$. The TRIzol ${ }^{\circledR}$ method was used to extract the total RNA from hepatic carcinoma tissues and cells according to the manufacturer's instructions (Invitrogen; Thermo Fisher Scientific, Inc.). The primers were designed using Primer 5 software (http://frodo.wi.mit.edu/cgi-bin/primer3/primer3 www.cgi) and synthesized by the Shanghai Yingwei Jieji Company. For cDNA synthesis, a $20 \mu 1$ reaction solution was prepared according to the reverse transcription kit (Roche Diagnostics). The amplification conditions of the thermal cycler were as follows: $25^{\circ} \mathrm{C}$ for $10 \mathrm{~min} ; 55^{\circ} \mathrm{C}$ for $30 \mathrm{~min}$; and $85^{\circ} \mathrm{C}$ for $5 \mathrm{sec}$. RT-qPCR was used to quantitatively detect the expression of each gene. A total of $2 \mu \mathrm{l}$ cDNA of each clinical specimen was obtained; then, $10 \mu \mathrm{l} \mathrm{SYBR}$ qPCR mix (Toyobo Life Science) and $2 \mu \mathrm{l}$ primer mixture were added, and $\mathrm{dH}_{2} \mathrm{O}$ was added to the total volume of $20 \mu \mathrm{l}$. The LightCycler ${ }^{\circledR} 480$ was used to detect relative expression. Gene expression was quantified using the $2^{-\Delta \Delta \mathrm{Cq}}$ method (18). The primer sequences were as follows: IncRNA-XIST forward, 5'-ACGCTGCAT GTGTCCTTAG-3'; IncRNA-XIST reverse, 5'-GAGCCTCTT ATAAGCTGTTTG-3'; miR-320a forward, 5'-CAACAGAAG GCTCGAGGGAAGTCTGCGTGGCAGG-3'; miR-320a reverse, 5'-ATTCTGATCAGGATCCGAGGCGAATCCTCA CATTG-3'; PIK3CA forward, 5'-GGTGACTGTGTGGGA CTTATTG-3'; PIK3CA reverse, 5'-TGATGTAGTGTGTGG CTGTTG-3'; GAPDH forward, 5'-CATCACCATCTTCCA GGAGCG-3'; GAPDH reverse, 5'-TGACCTTGCCCACAG CCTTG-3'; U6 forward, 5'-CTCGCTTCGGCAGCACA-3'; U6 reverse, 5'-AACGCTTCACGAATTTGCGT-3'.

Cell count assay. Following treatment, the cells in each group were trypsinized and collected, centrifuged at $300 \times \mathrm{g}\left(4^{\circ} \mathrm{C}\right)$ for $5 \mathrm{~min}$ and the supernatant was removed, and the complete medium was added to adjust the cell concentration to $5 \times 10^{3} / \mathrm{ml}$. 
The cells were seeded in a 96-well plate at $100 \mu \mathrm{l}$ cells per well, with three replicate wells. After culturing for 12, 24, 48 and $72 \mathrm{~h}$, a total of $10 \mu \mathrm{l} \mathrm{Cell} \mathrm{Counting} \mathrm{Kit} \mathrm{(CCK)-8} \mathrm{reagent}$ (Beyotime Institute of Biotechnology) was added to each well, and incubated at $37^{\circ} \mathrm{C}$ for $1.5 \mathrm{~h}$ in the incubator. A microplate reader was used to determine the absorbance value of each well; the wavelength was $450 \mathrm{~nm}$, and the proliferation curve was drawn. The cell count assay is an indirect form that can be used to measure cell proliferation.

Flow cytometry for cell apoptosis. According to the instructions of the apoptosis kit (Dojindo Molecular Technologies, Inc.), the cells in each group were trypsinized and collected and washed twice with phosphate buffered saline (PBS). The cells were centrifuged at $300 \mathrm{x}$ g for $5 \mathrm{~min}\left(4^{\circ} \mathrm{C}\right)$ and then $100 \mu \mathrm{l}$ Annexin $\mathrm{V}$ buffer was added to suspend the cells. Finally, PI and Annexin V staining solutions were added separately and mixed by pipetting. After $15 \mathrm{~min}$ of staining at room temperature and in the dark, $400 \mu \mathrm{l}$ Annexin V buffer was added to the sample, and cell apoptosis was detected by flow cytometry.

Transwell assay. After the cells from each group were digested with trypsin, the cell concentration was adjusted to $1 \times 10^{8} / 1$. Matrigel on the Transwell membrane was used to detect cell invasion ability, but Matrigel was not required to measure cell migration ability. A total of $100 \mu \mathrm{l}$ DMEM without FBS was added to the upper chamber of each Transwell, while $500 \mu \mathrm{l}$ of DMEM was added to the lower chamber. The Transwell chamber was incubated in an incubator with $5 \% \mathrm{CO}_{2}$ at $37^{\circ} \mathrm{C}$ for $24 \mathrm{~h}$. The cells were fixed with $4 \%$ paraformaldehyde for $15 \mathrm{~min}$ at room temperature, and washed thrice with PBS. Then, the cells were stained with $1 \%$ crystal violet at room temperature for $10 \mathrm{~min}$. After washing away the crystal violet staining solution, cell migration and invasion were observed under a light microscope (Leica Microsystems $\mathrm{GmbH}$ ). Ten fields were selected to calculate the average number of cells that migrated and invaded with a magnification of $x 400$.

Luciferase reporter experiment. The binding regions of lncRNA XIST and PIK3CA with miR-320a were predicted based on the StarBase (http://starbase.sysu.edu.cn/) and TargetScan databases (http://www.targetscan.org/vert_72/). Wild-type lncRNA XIST (lncRNA XIST-wt), wild-type PIK3CA non-coding region (PIK3CA-WT), mutant lncRNA XIST (lncRNA XIST-Mut) sequence, and mutant PIK3CA (PIK3CA-Mut) missing the miR-320a binding region, as well as mutations missing the miR-320a binding region were designed and the luciferase reporter vector was constructed by Nanjing Cobioer Gene Technology Co., Ltd. The lncRNA XIST-wt, IncRNA XIST-Mut, PIK3CA-WT and PIK3CA-Mut luciferase reporter vectors were transfected into the control and experimental cells at $37^{\circ} \mathrm{C}$ for $48 \mathrm{~h}$ (using Lipofectamine ${ }^{\circledR}$ 2000), and the Promega dual-luciferase reporter gene kit (Promega Corporation) was used to test the luciferase activity after transfection. Fluorescent enzyme activity was recorded as the ratio of firefly luciferase to Renilla luciferase activity.

Pull-down assay. The RNA pull-down experiment was performed according to the instructions of the RNA pull-down kit (Thermo Fisher Scientific, Inc.). The brief steps were as follows: The labeled target RNA was combined with streptavidin magnetic beads, and 30 Ult of streptavidin magnetic beads were added to a $1.5-\mathrm{ml}$ centrifuge tube. The magnetic beads were collected, and an equal volume of RNA capture buffer was used for resuspension and gentle pipetting. A total of 50 pmol biotin-labeled RNA was added, mixed well, and incubated for $30 \mathrm{~min}$ at room temperature. The magnetic beads were collected and $100 \mu \mathrm{l}$ RNA binding buffer was added and mixed. Then, the liquid was discarded and $100 \mu \mathrm{l}$ of the mixed RNA binding reaction solution was combined with the magnetic beads. Elution buffers (50 $\mu \mathrm{l})$ were used to elute the complex. The eluate was collected, RNA was extracted with TRIzol ${ }^{\circledR}$ and stored at $-80^{\circ} \mathrm{C}$, and the amount of enriched miR-320a was detected.

Western blot analysis. Western blotting was performed in accordance with conventional methods. Protein extraction buffer (Shanghai Kanglang Biotechnology Co., Ltd.) was used to extract the total protein of each group of hepatocellular carcinoma cells. The concentration of each histone was detected by the bicinchoninic acid (BCA) protein quantitative method, and all proteins were boiled and denatured at $100^{\circ} \mathrm{C}$. A total of $50 \mu \mathrm{g}$ of each protein group was subjected to $10 \%$ acrylamide gel electrophoresis, and transferred to a polyvinylidene fluoride membrane at a constant current. The membrane was sealed in 5\% skim milk at room temperature for $1 \mathrm{~h}$, washed with PBS, and incubated with the primary antibody PIK3CA (1:1,000; cat. no. ab40776; Abcam) at $4^{\circ} \mathrm{C}$ overnight. After washing with TPBS, the membrane was incubated with Alexa Fluor ${ }^{\circledR} 488$ goat anti-rabbit IgG (H+L) (ab150077; 1:2,000; cat. no. ab150077; Abcam) at room temperature for $1 \mathrm{~h}$. Finally, the protein bands were illuminated, developed and fixed by enhanced chemiluminescence (Pierce; Thermo Fisher Scientific Inc.). Signals were quantified using ImageJ 1.63 software (National Institutes of Health).

Statistical analysis. For any quantified parameter, each group had three parallels, and the experiment was repeated three times. SPSS 21.0 (IBM Corp.) was the statistical software used for the data analysis. The data are represented by the mean \pm standard deviation. Two-way ANOVA (two-way analysis of variance) with post hoc test was used. Kaplan-Meier survival analysis was applied to examine the association between gene expression and survival rate, and statistical significance was assessed using the Mantel-Cox log rank test. Pearson's correlation was used to test the gene expression correlation. $\mathrm{P}<0.05$ was used to indicate a statistically significant difference.

\section{Results}

IncRNA XIST expression is higher in hepatic carcinoma tissues and cells. RT-qPCR was used to detect the expression level of lncRNA XIST in hepatic carcinoma tissues and cells. Compared with normal tissues, lncRNA XIST was highly expressed in hepatic carcinoma tissues $(\mathrm{P}<0.01$; Fig. 1A). Moreover, with increasing tumor stage, the expression of lncRNA XIST also increased (Fig. 1B). Based on the median value of IncRNA XIST expression (cut-off value, 1.815), the 69 patients were divided into the highly expressed lncRNA 

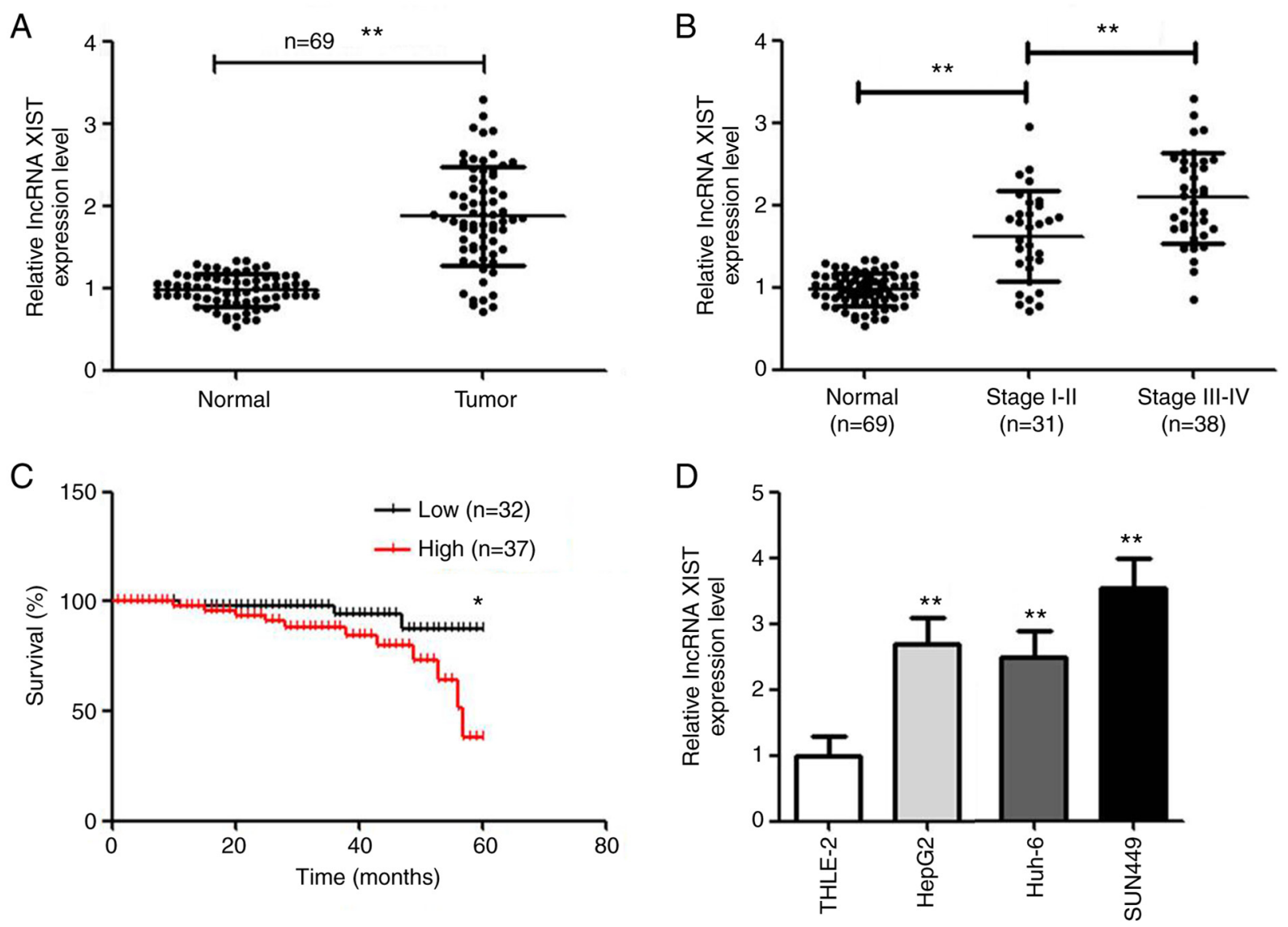

Figure 1. lncRNA XIST is highly expressed in hepatic carcinoma cells and tissues. (A) The reverse transcription-quantitative PCR detection of lncRNA XIST expression in 69 pairs of hepatic carcinoma tissues. ${ }^{* *} \mathrm{P}<0.01$ vs. normal group. (B) Expression level of lncRNA XIST in different graded tissues. ${ }^{* *} \mathrm{P}<0.01$ vs. stage I-II. (C) Survival curve analysis. ${ }^{*} \mathrm{P}<0.05$ and ${ }^{* *} \mathrm{P}<0.01$ vs. normal group. (D) LncRNA XIST was highly expressed in hepatic carcinoma cell lines compared with THLE- 2 cells. ${ }^{* *} \mathrm{P}<0.01$ vs. normal group. lncRNA, long non-coding RNA.

XIST group $(\mathrm{n}=37)$ and low-expressed lncRNA XIST group $(\mathrm{n}=32)$. As shown in Fig. 1C, the survival rate was significantly lower in the highly expressed lncRNA XIST group. Moreover, tumor size, vascular invasion and classification were significantly different between the two groups (Table I). The lncRNA XIST was highly expressed in all hepatic carcinoma cells compared with the THLE-2 cell line (Fig. 1D). Among the hepatic carcinoma cell lines, lncRNA XIST was expressed the highest in the HepG2 and SUN449 cell lines. Thus, the HepG2 and SUN449 cell lines were used in the following experiments.

shlncRNAXIST inhibits the proliferation, invasion and migration of hepatic carcinoma cells. After 3 shlncRNA XIST transfection, the transfection efficiency was detected. In terms of the results, the relative lncRNA XIST level was significantly decreased, and shlncRNA XIST-1 was associated with the highest transfection efficiency (Fig. 2A). For the following experiments, shlncRNA XIST-1 was used and defined as shlncRNA XIST. Following shlncRNA XIST transfection for $72 \mathrm{~h}$, the proliferation of both HepG2 and SUN449 cell lines significantly decreased (Fig. 2B). Moreover, the apoptosis rate was significantly higher in the shlncRNA XIST group in both cell lines (Fig. 2C). The invasion and migration of hepatic carcinoma cells were also inhibited by shlncRNA XIST (Fig. 2D and E). Thus, shlncRNA XIST inhibited cell proliferation, invasion and migration, while promoting the apoptosis of hepatic carcinoma cells.
IncRNA XIST negatively regulates miR-320a. Starbase was applied to predict the targeting association between lncRNA XIST and miR-320a. The binding site is shown in Fig. 3A. In the IncRNA XIST-WT group, the miR-320a mimic resulted in significantly decreased luciferase activity, while the miR-320a inhibitor increased its activity. However, both the miR-320a mimic and miR-320a inhibitor had no effect on luciferase activity in the IncRNA XIST-MUT group (Fig. 3B). Compared with the empty vectors and mutants, biotin-labeled lncRNA XIST was used to pull down miR-320a by affinity in vitro, and the results confirmed the endogenous binding between miR-320a and lncRNA XIST (Fig. 3C). In addition, the expression level of miR-320a was also detected in hepatocellular carcinoma cells and tissues. As shown in Fig. 3D and E, the miR-320a demonstrated a lower expression level in hepatocellular carcinoma cells and tissues. Pearson's correlation analysis also confirmed that miR-320a and lncRNA XIST demonstrated a negative association in patients with hepatocellular carcinoma (Fig. 3F). Notably, the relative miR-320a expression level was significantly increased following shlncRNA XIST transfection (Fig. 3G).

miR-320a negatively targets PIK3CA. The Starbase online tool was used to predict the targeting association between PIK3CA and miR-320a. The binding site of PIK3CA and miR-320a is shown in Fig. 4A. miR-320a mimic, miR-320a inhibitor and miR-NC were transfected in cells; the transfections were successful (Fig. 4B). 
Table I. Association between long non-coding RNA XIST expression and general clinical parameters.

\begin{tabular}{|c|c|c|c|c|}
\hline \multirow[b]{2}{*}{ Characteristics } & \multirow[b]{2}{*}{ Number of patients } & \multicolumn{2}{|c|}{ lncRNA XIST } & \multirow[b]{2}{*}{ P-value } \\
\hline & & Low expression (< median) & High expression ( $\geq$ median) & \\
\hline Number & 40 & 20 & 20 & \\
\hline Sex & & & & 0.342 \\
\hline Male & 19 & 11 & 8 & \\
\hline Female & 21 & 9 & 12 & \\
\hline Age, years & & & & 0.337 \\
\hline$<60$ & 23 & 13 & 10 & \\
\hline$\geq 60$ & 17 & 7 & 10 & \\
\hline Tumor size, cm & & & & 0.008 \\
\hline$<5$ & 26 & 17 & 9 & \\
\hline$\geq 5$ & 14 & 3 & 11 & \\
\hline Tumor site & & & & 0.311 \\
\hline Colon & 13 & 5 & 8 & \\
\hline Rectum & 27 & 15 & 12 & \\
\hline Depth of invasion & & & & 0.168 \\
\hline $\mathrm{T} 1-\mathrm{T} 2$ & 12 & 8 & 4 & \\
\hline T3-T4 & 28 & 12 & 16 & \\
\hline Lymph node metastasis & & & & 0.011 \\
\hline N0 & 22 & 15 & 7 & \\
\hline $\mathrm{N} 1-\mathrm{N} 2$ & 18 & 5 & 13 & \\
\hline Distant metastasis & & & & 0.212 \\
\hline M0 & 33 & 18 & 15 & \\
\hline M1 & 7 & 2 & 5 & \\
\hline TNM stage & & & & 0.004 \\
\hline I-II & 19 & 14 & 5 & \\
\hline III-IV & 21 & 6 & 15 & \\
\hline
\end{tabular}

TNM, Tumor-Node-Metastasis.

The luciferase reporter gene experiment was undertaken to verify whether binding occurred. In the PIK3CA-WT group, the miR-320a mimic significantly decreased the luciferase activity, while the miR-320a inhibitor increased the activity. However, both the miR-320a mimic and miR-320a inhibitor had no effect on luciferase activity in the PIK3CA-MUT group (Fig. 4C). The expression of PIK3CA in hepatocellular carcinoma cells was detected by RT-qPCR. As shown in Fig. 4D, the level of PIK3CA was significantly higher in hepatocellular carcinoma cells compared with the THLE-2 cell line. In hepatocellular carcinoma and normal tissues, the expression of PIK3CA exhibited a similar trend (Fig. 4E). Following transfection with miR-320 mimic, the expression level of PIK3CA significantly decreased (Fig. 4F). Pearson's correlation analysis was used to confirm the correlation between PIK3CA and lncRNA XIST or miR-320a expression. The results showed that PIK3CA was negatively correlated with miR-320a, while PIK3CA was positively correlated with IncRNA XIST (Fig. 4G and H).

miR-320a mimic and PIK3CA inhibitor rescues the effect of oelncRNA XIST. The rescue assay was performed in SUN449 cell lines. Small interfering RNA (si)PIK3CA significantly decreased PIK3CA expression, indicating the transfection was successful(Fig.5A). Based on transfection, the cells were divided into MOCK, oelncRNA XIST, oelncRNA XIST+miR-320a mimic and oelncRNA XIST+PIK3CA inhibitor groups. After transfection, oelncRNA XIST significantly increased lncRNA XIST expression level (Fig. 5B). OelncRNA XIST significantly increased PIK3CA, while miR-320a mimic and PIK3CA inhibitor decreased the expression of PIK3CA at the gene and protein level (Fig. 5C). With respect to the effects of miR-320a, PIK3CA and lncRNA XIST levels on hepatocellular carcinoma development, CCK-8, flow cytometry and the Transwell assay were processed. When examining the results, oelncRNA XIST increased the cell number, invasion and migration, while it decreased the apoptosis of SUN449 cells. The cell number is an indirect measure of proliferation. More importantly, the miR-320a mimic and PIK3CA inhibitor demonstrated the recovery effects of oelncRNA XIST in the aforementioned processes (Fig. 5D-G). The aforementioned findings indicate that IncRNA XIST accelerates hepatic carcinoma progression by targeting the miR-320a/PIK3CA axis. 

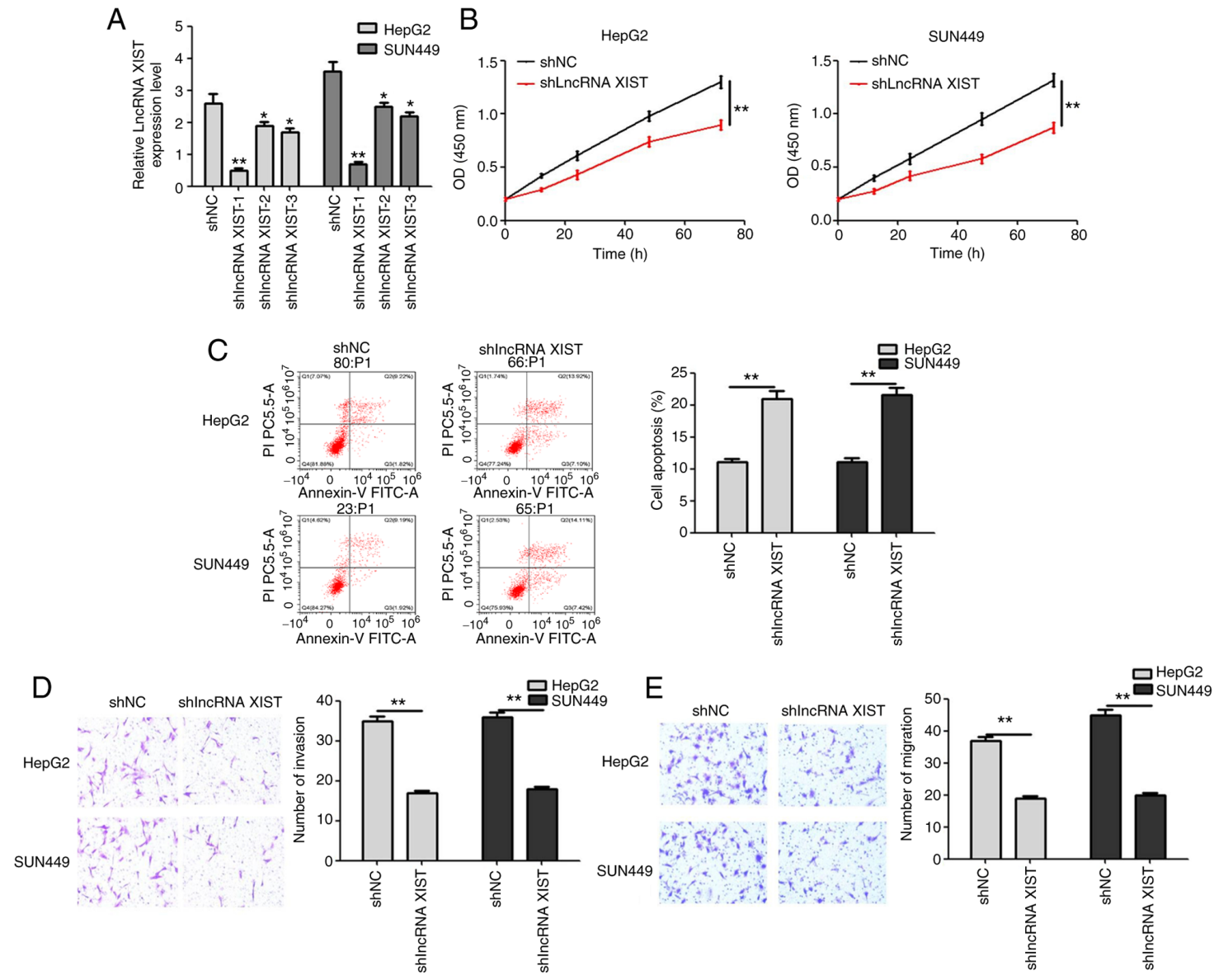

Figure 2. shlncRNA XIST inhibits the cell number, invasion and migration of hepatic carcinoma cells. (A) Reverse transcription-quantitative PCR was used to detect the expression of lncRNA XIST after shlncRNA XIST-1, shlncRNA XIST-2 and shlncRNA XIST-3 transfections. (B) Cell Counting Kit-8 assay to count the number of hepatic carcinoma cells after shlncRNA XIST transfection. (C) Flow cytometry to detect apoptosis after shlncRNA XIST transfection. (D) Transwell assay was processed to detect the invasion ability after shlncRNA XIST transfection; magnification, $\mathrm{x} 400$ ). (E) Transwell assay was undertaken for migration ability after shlncRNA XIST transfection; magnification, $\mathrm{x} 400),{ }^{*} \mathrm{P}<0.05$ and ${ }^{* *} \mathrm{P}<0.01$ vs. sh-NC. sh, short hairpin RNA; lncRNA, long non-coding RNA; NC, negative control.

\section{Discussion}

Hepatocellular carcinoma is the most common primary malignant liver tumor (19). Due to its high incidence rate, early metastasis and postoperative recurrence, the patient survival rate is low (20). At present, there are few effective diagnostic markers of hepatocellular carcinoma. In the present study, lncRNA XIST was found to be highly expressed in hepatic carcinoma tissues and cells. Moreover, the expression of lncRNA XIST was closely associated with disease stage and the patient survival rate. In order to provide a potential biomarker to determine the prognosis of hepatic carcinomas, the molecular mechanism of IncRNA XIST was explored in the present study.

LncRNA XIST was the first non-coding gene identified within the X inactivation center (XIC) (21). In previous studies, it has been confirmed to regulate various cancer types, such as gastric cancer, osteosarcoma, colorectal cancer and non-small cell lung cancer (22-25). Moreover, IncRNA XIST participated in different pathways, including the DNA mismatch repair, NF- $\kappa B / N L R P 3$ inflammasome, HIF-1A/AXL signaling and iASPP pathways $(13,26-28)$. Thus, the DNA mismatch repair defects are involved in a variety of gastrointestinal and other tumors. In hepatic carcinoma, there is a loss of heterozygosity at the hMSH2 and/or hMLH1 gene locus, as well as the instability of associated microsatellites in liver tissues prior to malignant transformation (29). Wu et al (30) found that inhibiting the activation of NF- $\mathrm{KB} / \mathrm{NLRP} 3$ inflammasomes could decrease the immune-inflammatory response, thereby preventing the disorder and accumulation of lipid metabolism in the liver under high-fructose conditions. It is worth noting that lncRNA XIST was found to interact with miR-497-5p and target programmed cell death 4 (PDCD4), and it was further involved in the migration and proliferation of hepatic cancer cells (16). Similar results were also obtained in the present study, as shlncRNA XIST attenuated the proliferation, invasion and migration of hepatic cancer cells, while it accelerated apoptosis.

Although lncRNA XIST does not encode proteins, it could still function at the RNA level to regulate gene expression. In the present study, IncRNA XIST negatively targeted miR-320a. miR-320a is a member of the miR-320 family, which is widely present in various tissues and organs of the human body. In recent years, many studies have found that the expression level of miR-320a was high in the stomach, liver, colorectal and other tumor cells, and it was also confirmed that miR-320a is closely associated with tumor occurrence, development, metastasis and prognosis $(31,32)$. 
A

Target: 5' ggGCCAGUCUUGAGCCAGCUUUa 3 ' miRNA : 3' agCGG-GAGAGUUGGGUCGAAAa 5 ,
$\mathrm{B}$

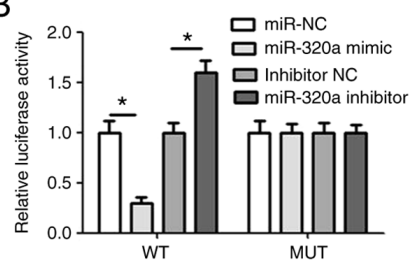

C

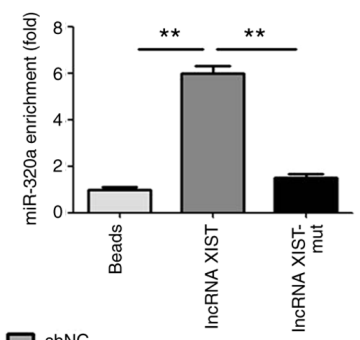

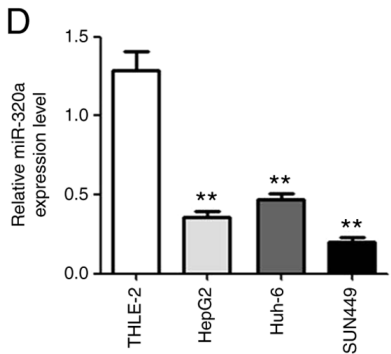

$\mathrm{F}$

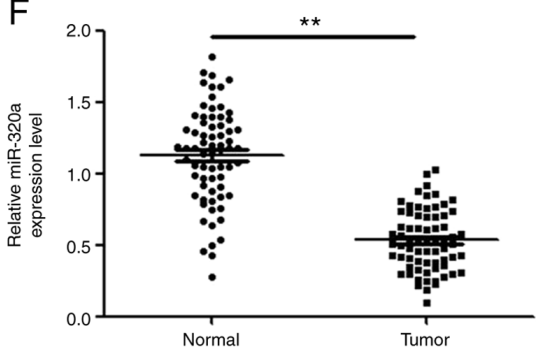

$\mathrm{E}$
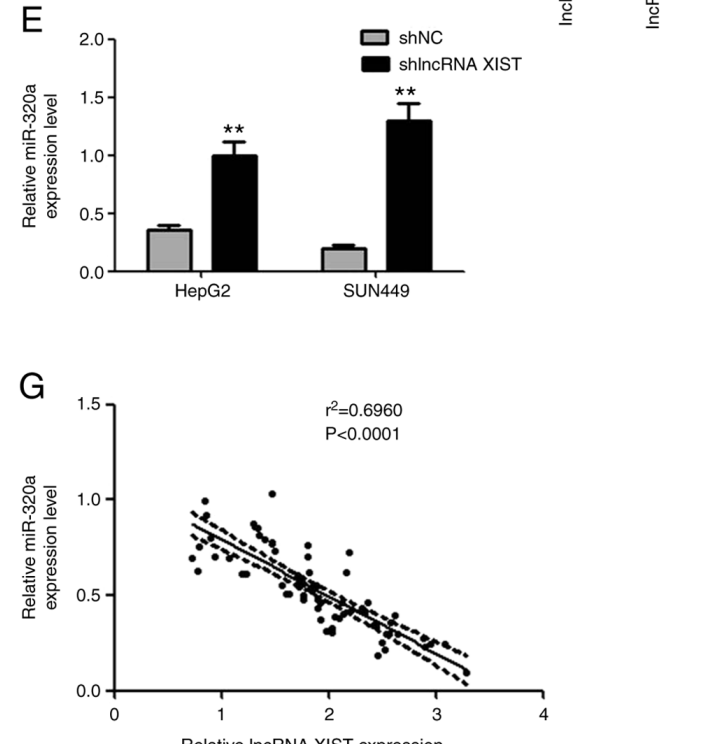

Figure 3. IncRNA XIST negatively regulates miR-320a. (A) The binding site of lncRNA XIST and miR-320a. (B) Luciferase reporter experiment to verify the binding of lncRNA XIST and miR-320a. (C) Pull-down assay. (D) The expression level of miR-320a in hepatocellular carcinoma cells. (E) The expression level of miR-320a in hepatocellular carcinoma tissues. (F) miR-320a and lncRNA XIST were negatively associated in patients with hepatocellular carcinoma. (G) The relative miR-320a expression level after shlncRNA XIST transfection. ${ }^{*} \mathrm{P}<0.05$ and ${ }^{* *} \mathrm{P}<0.01$ vs. the normal or NC group. lncRNA, long non-coding RNA; miR/miRNA, microRNA; NC, negative control; WT, wild type; MUT, mutant; sh, short hairpin RNA.

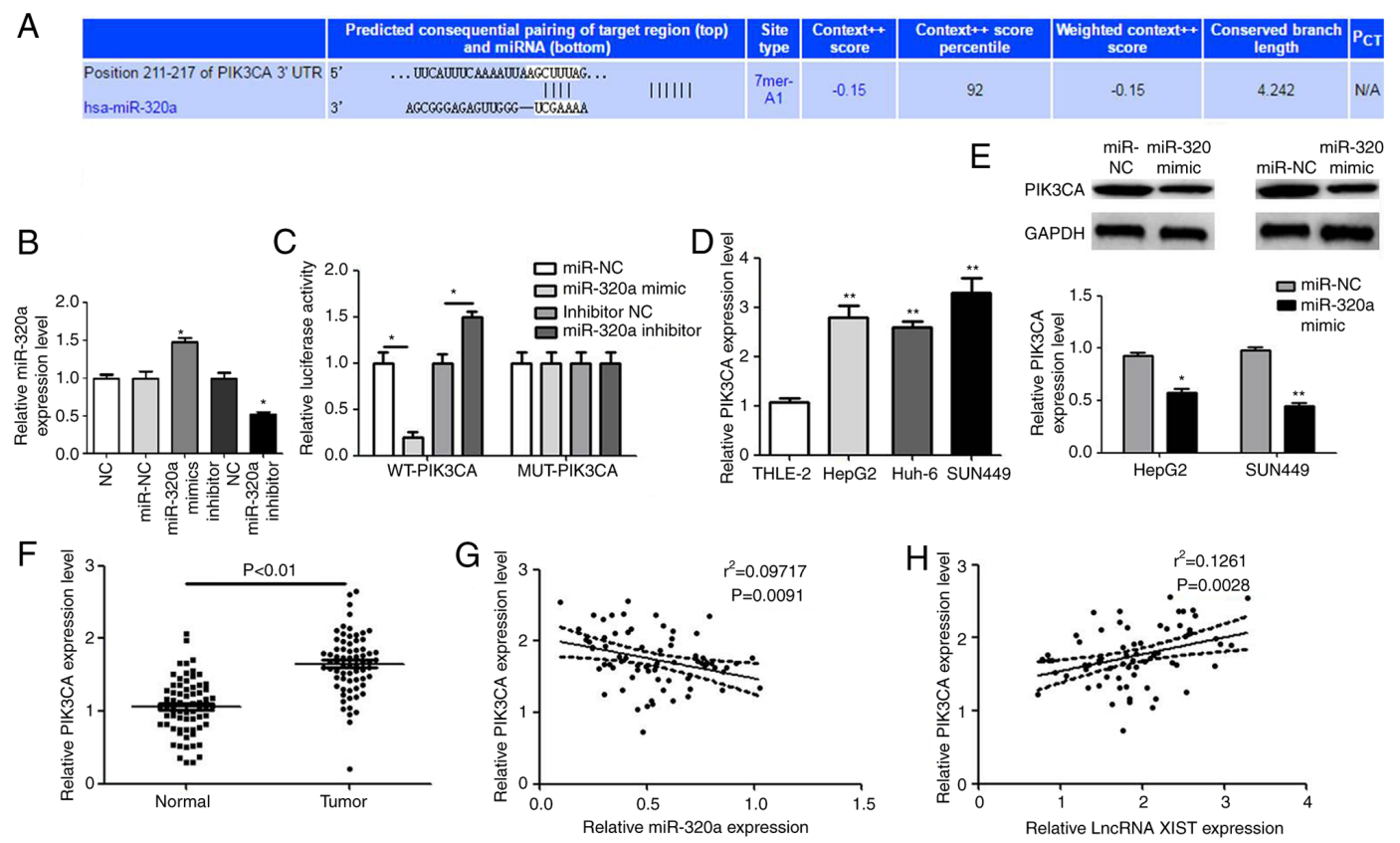

Figure 4. miR-320a negatively targets PIK3CA. (A) The binding site of PIK3CA and miR-320a, as based on the Targetscan database. (B) Transfection efficiency of miR-320a mimic, inhibitor and NC. "P<0.05 vs. NC group. (C) Fluorescent reporter assay to verify the binding of PIK3CA and miR-320a. "P<0.05 miR-320a mimic vs. miR-320a NC group, ${ }^{*} \mathrm{P}<0.05$ miR-320a inhibitor vs. inhibitor NC group. (D) The expression of PIK3CA in hepatocellular carcinoma cells. ${ }^{*} \mathrm{P}<0.05$ vs. THLE-21 group. (E) The effect of miR-320a on PIK3CA levels. "P<0.05 and ${ }^{* *} \mathrm{P}<0.01$ vs miR-NC group. (F) The expression of PIK3CA in hepatocellular carcinoma and normal tissues. The expression correlation between (G) PIK3CA and miR-320a and (H) between PIK3CA and lncRNA XIST, Compared with NC or normal group, ${ }^{*} \mathrm{P}<0.05$ vs. NC or THLE-21 group, ${ }^{* *} \mathrm{P}<0.01$. miR, microRNA; NC, negative control; lncRNA, long non-coding RNA; WT, wild type; MUT, mutant; UT3, untranslated region. 

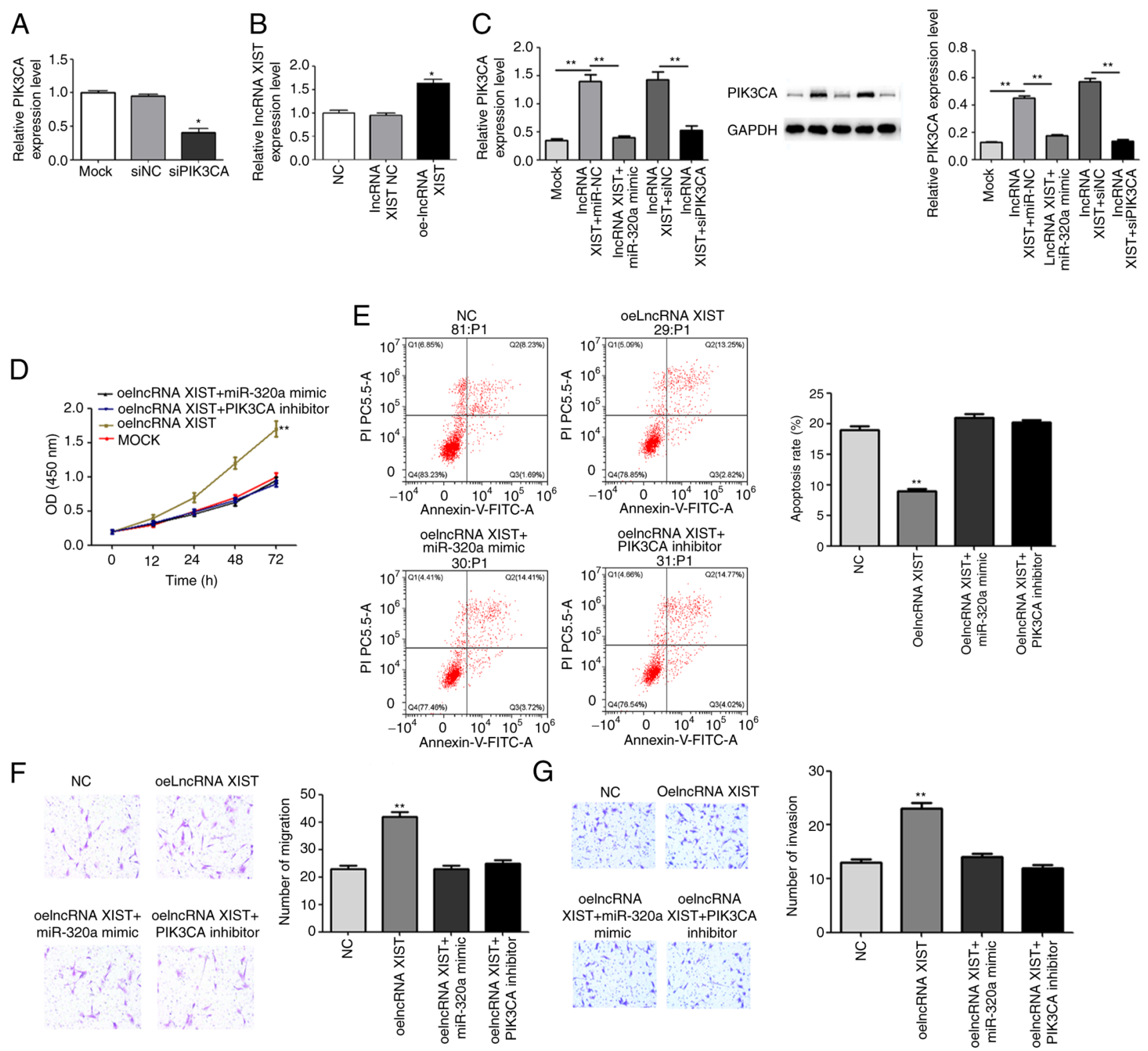

G
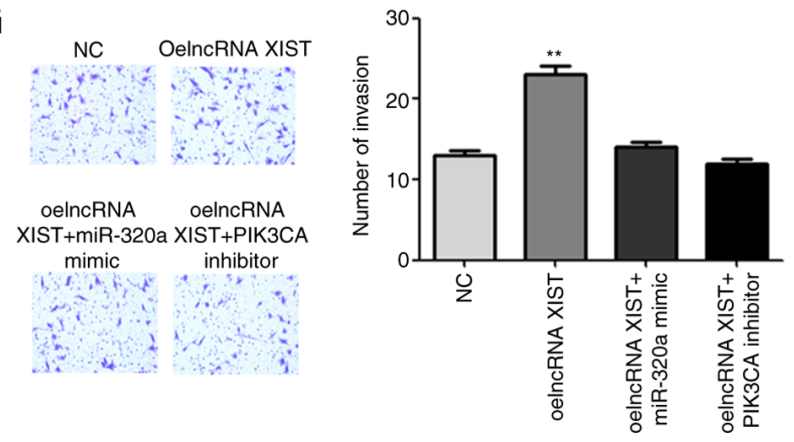

Figure 5. miR-320a mimic and PIK3CA inhibitor recover the effect of oelncRNA XIST. The rescue assay was performed in the SUN449 cell line. Based on transfection, the cells were divided into the MOCK, oelncRNA XIST, oelncRNA XIST+miR-320a mimic and oelncRNA XIST+PIK3CA inhibitor groups. (A) Transfection efficiency of siPIK3CA. (B) Transfection efficiency of oelncRNA XIST. (C) The relative PIK3CA expression in each group was detected using reverse transcription-quantitative PCR and western blotting. (D) The Cell Counting Kit-8 assay was used to measure proliferation. (E) Flow cytometry for apoptosis. (F) Transwell assay to assess invasion; magnification, $\mathrm{x} 400$. (G) Transwell assay for migration assay; magnification, $\mathrm{x} 400$, $\mathrm{P}<0.05$ and ${ }^{* * *} \mathrm{P}<0.01$ vs. NC or mock group. miR, microRNA; oe, overexpression vector; lncRNA, long non-coding RNA; si, small, interfering RNA; NC, negative control.

Wen et al (33) detected the expression of miRNA in the blood and tissues of patients with hepatocellular carcinoma, and found that the expression of miR-320a was significantly increased. The aforementioned findings further confirmed that miR-320a could be used as a biological indicator for the early detection of hepatocellular carcinoma. Additionally, Yao et al (34) investigated the biological functions of miRNA in hepatocellular carcinoma cells, and found that the overexpression of miR-320a significantly enhanced the migration and invasion of hepatocellular carcinoma cells, while downregulating miR-320a inhibited the cells' migration and invasion ability. It may be that miR-320a decreased the expression of the G protein subunit alpha I1 (GNAI1), thereby promoting the metastasis and invasion of tumor cells. Besides, miR-320a might participate in the epithelial-mesenchymal transition pathway, and affect the progression of hepatocellular carcinoma (35). In SMMC-7221 cell lines, miR-320a was also found to have a lower expression compared with normal cells, which further influenced migration and apoptosis (36). Interestingly, the miR-320a mimic recovered the effect of lncRNA XIST in the present study. Based on the aforementioned findings, IncRNA XIST might promote hepatic carcinoma progression by regulating miR-320a expression.

The results in the present study also confirmed that miR-320a negatively targeted PIK3CA. PIK3CA is a phosphatidylinositol kinase that phosphorylates the third hydroxyl group of the inositol ring (37). PIK3CA activation 
was associated with the production of the second messenger PIP3 on the plasma membrane. PIP3 bound to the signal proteins AKT serine/threonine kinase (AKT) and pyruvate dehydrogenase kinase 1 (PDK1), containing the PH domain in the cell. This prompted PDK1 to phosphorylate the AKT protein Set308, leading to the activation of AKT $(38,39)$. Activated AKT promoted or inhibited its downstream target proteins (Bad, Caspase, NF-KB, GSK-3 and FKHR) through phosphorylation, thereby regulating cell proliferation, differentiation, apoptosis and migration (40). In addition, the PIK3CA inhibitor could recover the effect of lncRNA XIST in the present study. As a result, lncRNA XIST accelerated hepatic carcinoma progression by regulating PIK3CA.

According to the obtained results, IncRNA XIST accelerates hepatic carcinoma progression by targeting the miR-320a/PIK3CA axis, which might serve as the theoretical basis underlying targeted therapy for hepatic carcinomas.

\section{Acknowledgements}

Not applicable.

\section{Funding}

No funding was received.

\section{Availability of data and materials}

The datasets used and/analyzed during the current study are available from the corresponding author on reasonable request.

\section{Authors' contributions}

LY, JJ and YZ made substantial contributions to the study design. FX, XT, WX and TX conducted the experiments. WX, YN and TX analyzed and interpreted the data. All authors have read and approved the final manuscript. LY and JJ confirm the authenticity of all the raw data.

\section{Ethics approval and consent to participate}

The study was approved by the ethics committee of Lianyungang No. 1 People's Hospital (permit no. LW-20140322001), which complied with medical ethics regulations. All patients signed an informed consent form.

\section{Patient consent for publication}

Not applicable.

\section{Competing interests}

The authors declare that they have no competing interests.

\section{References}

1. Li B, Liu H, Shang HW, Li P, Li N and Ding HG: Diagnostic value of glypican-3 in alpha fetoprotein negative hepatocellular carcinoma patients. Afr Health Sci 13: 703-709, 2013.

2. Akoad ME and Pomfret EA: Surgical resection and liver transplantation for hepatocellular carcinoma. Clin Liver Dis 19: 381-399, 2015
3. Yamamoto J, Kosuge T, Saiura A, Sakamoto Y, Shimada K, Sano T, Takayama T, Sugawara Y, Yamaguchi T, Kokudo N and Makuuchi M: Effectiveness of hepatic resection for early-stage hepatocellular carcinoma in cirrhotic patients: Subgroup analysis according to Milan criteria. Jpn J Clin Oncol 37: 287-295, 2007.

4. Itoh A, Sadamori H, Yabushita K, Monden K, Tatsukawa M, Hioki M, Hyodo T, Omonishi K, Ueki T, Ohno S, et al: Advanced hepatocellular carcinoma with hepatic vein tumor thrombosis and renal dysfunction after hepatic arterial infusion chemotherapy effectively treated by liver resection with active veno-venous bypass: Report of a case. BMC Cancer 16: 705, 2016.

5. Lotersztajn S, Julien B, Teixeira-Clerc F, Grenard P and Mallat A: Hepatic fibrosis: Molecular mechanisms and drug targets. Annu Rev Pharmacol Toxicol 45: 605-628, 2005.

6. Huang X, Zhi X, Gao Y, Na T and Zheng J: LncRNAs in pancreatic cancer. Oncotarget 7: 57379-57390, 2016.

7. Xu YY and Song X: Advances in research on circulating miRNAs and lncRNAs in early diagnosis and treatment of lung cancer. Tumor 35: 584-591, 2015 (In Chinese).

8. Deng L, Yang SB, Xu FF and Zhang JH: Long noncoding RNA CCAT1 promotes hepatocellular carcinoma progression by functioning as let-7 sponge. J Exp Clin Cancer Res 34: 18, 2015.

9. Zamani M, Sadeghizadeh M, Behmanesh M and Najafi F: Dendrosomal curcumin increases expression of the long non-coding RNA gene MEG3 via up-regulation of epi-miRs in hepatocellular cancer. Phytomedicine 22: 961-967, 2015.

10. Li T, Xie J, Shen C, Cheng D, Shi Y, Wu Z, Deng X, Chen H, Shen B, Peng C, et al: Amplification of long noncoding RNA ZFAS1 promotes metastasis in hepatocellular carcinoma. Cancer Res 75: 3181-3191, 2015.

11. Tsang WP and Kwok TT: Riboregulator H19 induction of MDR1-associated drug resistance in human hepatocellular carcinoma cells. Oncogene 26: 4877-4881, 2007.

12. Du Y, Weng XD, Wang L, Liu XH, Zhu HC, Guo J, Ning JZ and Xiao CC: LncRNA XIST acts as a tumor suppressor in prostate cancer through sponging miR-23a to modulate RKIP expression. Oncotarget 8: 94358-94370, 2017.

13. Tang Y, He R, An J, Deng P, Huang L and Yang W: lncRNA XIST interacts with miR-140 to modulate lung cancer growth by targeting iASPP. Oncol Rep 38: 941-948, 2017.

14. Schouten PC, Vollebergh MA, Opdam M, Jonkers M, Loden M, Wesseling J, Hauptmann M and Linn SC: High XIST and low 53BP1 expression predict poor outcome after high-dose alkylating chemotherapy in patients with a BRCA1-like breast cancer. Mol Cancer Ther 15: 190-198, 2016.

15. Fang J, Sun CC and Gong C: Long noncoding RNA XIST acts as an oncogene in non-small cell lung cancer by epigenetically repressing KLF2 expression. Biochem Biophys Res Commun 478: 811-817, 2016.

16. Zhang Y, Zhu Z, Huang S, Zhao Q, Huang C, Tang Y, Sun C, Zhang Z, Wang L, Chen $\mathrm{H}$, et al: IncRNA XIST regulates proliferation and migration of hepatocellular carcinoma cells by acting as miR-497-5p molecular sponge and targeting PDCD4. Cancer Cell Int 19: 198, 2019

17. Kong Q, Zhang S, Liang C, Zhang Y, Kong Q, Chen S, Qin J and Jin Y: LncRNA XIST functions as a molecular sponge of miR-194-5p to regulate MAPK1 expression in hepatocellular carcinoma cell. J Cell Biochem 119: 4458-4468, 2018

18. Nolan T, Hands RE and Bustin SA: Quantification of mRNA using real-time RT-PCR. Nat Protoc 1: 1559-1582, 2006.

19. Inagawa S, Itabashi $M$, Adachi S, Kawamoto $T$, Hori $M$, Shimazaki J, Yoshimi F and Fukao K: Expression and prognostic roles of beta-catenin in hepatocellular carcinoma: Correlation with tumor progression and postoperative survival. Clin Cancer Res 8: 450-456, 2002

20. Li YT, Luo SF and Liu JF: Advances in research on prediction for early postoperative metastasis and recurrence in patients with hepatocellular carcinoma. Chin Med Abstracts 13: 259-261, 2013.

21. Lee JT: X-Chromosome Inactivation and Long Non-Coding RNAs. Presentation at the Annual Meeting of the American Association for the Advancement of Science Feb 13-17 2014 in Chicago, IL, USA.

22. Li J, Shi Z, Luo TH, Nie MM, Yuan SJ and Bi JW: Expression and clinical significance of long non-coding RNA XIST in tissue and plasma of gastric cancer. Acad J Sec Mil Med Univ 38: 1403-1409, 2017 (In Chinese).

23. Sun X, Wei B, Peng ZH, Fu QL, Wang CJ, Zheng JC and Sun JC: Knockdown of lncRNA XIST suppresses osteosarcoma progression by inactivating AKT/mTOR signaling pathway by sponging miR-375-3p. Int J Clin Exp Pathol 12: 1507-1517, 2019. 
24. Song H, He P, Shao T, Li Y, Li J and Zhang Y: Long non-coding RNA XIST functions as an oncogene in human colorectal cancer by targeting miR-132-3p. J BUON 22: 696-703, 2017.

25. Zhang YL, Li XB, Hou YX, Fang NZ, You JC and Zhou QH: The lncRNA XIST exhibits oncogenic properties via regulation of miR-449a and Bcl-2 in human non-small cell lung cancer. Acta Pharmacol Sin 38: 371-381, 2017.

26. Du P, Zhao H, Peng R, Liu Q, Yuan J, Peng G and Liao Y: LncRNA-XIST interacts with miR-29c to modulate the chemoresistance of glioma cell to TMZ through DNA mismatch repair pathway. Biosci Rep 37: BSR20170696, 2017.

27. Ma M, Pei Y, Wang X, Feng J, Zhang Y and Gao MQ: LncRNA XIST mediates bovine mammary epithelial cell inflammatory response via NF- $\mathrm{B} / \mathrm{NLRP} 3$ inflammasome pathway. Cell Prolif 52: e12525, 2019.

28. Yang LG, Cao MZ, Zhang J, Li XY and Sun QL: LncRNA XIST modulates HIF-1A/AXL signaling pathway by inhibiting miR-93-5p in colorectal cancer. Mol Genet Genomic Med 8 : e1112, 2020.

29. Macdonald GA, Greenson JK, Saito K, Cherian SP, Appelman HD and Boland CR: Microsatellite instability and loss of heterozygosity at DNA mismatch repair gene loci occurs during hepatic carcinogenesis. Hepatology 28: 90-97, 1998.

30. Wu Q, Meng WY, Jie Y and Zhao H: LncRNA MALAT1 induces colon cancer development by regulating miR-129-5p/HMGB1 axis. J Cell Physiol 233: 6750-6757, 2017.

31. Lin Y, Zeng Y, Zhang F, Xue L, Huang Z, Li W and Guo M: Characterization of MicroRNA expression profiles and the discovery of novel MicroRNAs involved in cancer during human embryonic development. PLoS One 8: e69230, 2013.

32. Zhang $\mathrm{B}$ and Pan X: RDX induces aberrant expression of MicroRNAs in Mouse brain and liver. Environ Health Perspect 117: 231-240, 2009.

33. Wen Y, Han J, Chen J, Dong J, Xia Y, Liu J, Jiang Y, Dai J, Lu J, Jin $\mathrm{G}$, et al: Plasma miRNAs as early biomarkers for detecting hepatocellular carcinoma. Int J Cancer 137: 1679-1690, 2015.
34. Yao J, Liang LH, Zhang Y, Ding J, Tian Q, Li JJ and He XH: GNAI1 suppresses tumor cell migration and invasion and is post-transcriptionally regulated by Mir-320a/c/d in hepatocellular carcinoma. Cancer Biol Med 9: 234-241, 2012.

35. Chou LF, Chen CY, Yang WH, Chen CC, Chang JL, Leu YL, Liou MJ and Wang TH: Suppression of hepatocellular carcinoma progression through FOXM1 and EMT inhibition via hydroxygenkwanin-induced miR-320a expression. Biomolecules 10: 20, 2019.

36. Lv X, Xiong W, Song D, Shi H, Li H, Guo P, Chen D and Li J: The expression of miR-320a on hepatocellular carcinoma and the effect of apoptosis and migration on SMMC-7721 cell line. Genomics Appl Biol 5: 2305-2311, 2018 (In Chinese).

37. Auger KR, Carpenter CL, Cantley LC and Varticovski L: Phosphatidylinositol 3-kinase and its novel product, phosphatidylinositol 3-phosphate, are present in Saccharomyces cerevisiae. J Biol Chem 264: 20181-20184, 1989.

38. Zhao JJ: Targeting breast cancers featuring activating mutations in PIK3CA by generating a lethal dose of PIP3. Annual summary rept. 1 Feb 2007-31 Jan 2008. Defense Technical Information Center, Fort Belvoir, VA, 2008. https://apps.dtic.mil/sti/citations/ ADA485990. Accessed February 1, 2008.

39. Orloff MS, He X, Peterson C, Chen F, Chen JL, Mester JL and Eng C: Germline PIK3CA and AKT1 mutations in Cowden and Cowden-like syndromes. Am J Hum Genet 92: 76-80, 2013.

40. Ali T, Badshah H, Kim TH and Kim MO: Melatonin attenuates D-galactose-induced memory impairment, neuroinflammation and neurodegeneration via RAGE/NF-K B/JNK signaling pathway in aging mouse model. J Pineal Res 58: 71-85, 2015.

This work is licensed under a Creative Commons Attribution-NonCommercial-NoDerivatives 4.0 International (CC BY-NC-ND 4.0) License. 